\title{
Vapour-Phase and Solid-Phase Epitaxy of Silicon on Solid-Phase Crystallised Seed Layers for Solar Cells Application
}

\author{
Wei Li, Sergey Varlamov, Miga Jung, and Jialiang Huang \\ School of Photovoltaic and Renewable Energy Engineering, University of New South Wales, Sydney, NSW 2052, Australia \\ Correspondence should be addressed to Wei Li; weili.unsw@gmail.com
}

Received 18 December 2013; Accepted 28 February 2014; Published 24 March 2014

Academic Editor: Man Shing Wong

Copyright ( 2014 Wei Li et al. This is an open access article distributed under the Creative Commons Attribution License, which permits unrestricted use, distribution, and reproduction in any medium, provided the original work is properly cited.

\begin{abstract}
Vapour-phase and solid-phase epitaxy are used for thickening of a solid-phase crystallised silicon seed layer on glass. Crosssectional transmission microscope images confirm that a transfer of crystallographic information has taken place from the seed layer into the epilayers. X-ray diffraction, scanning electron microscopy, and transmission electron microscopy reveal that the density of planar defects (mainly on $\{111\}$ plains) in the vapour-phase epitaxial sample is much higher than in the solid-phase epitaxial sample. These planar defects can act as recombination centres for free-charge carriers. Consequently, PC1D modelled minority carrier diffusion length in the vapour-phase grown epilayer is $50 \%$ shorter than that in the solid-phase grown epilayer. As a result, a solar cell grown by solid-phase epitaxy achieves open circuit voltage of $468 \mathrm{mV}$, short circuit current of $9.17 \mathrm{~mA} / \mathrm{cm}^{2}$, and photovoltaic conversion efficiency at $2.75 \%$ which are all higher than those of the solar cell grown by vapour-phase epitaxy on the same seed layer, $400 \mathrm{mV}, 7.28 \mathrm{~mA} / \mathrm{cm}^{2}, 1.69 \%$, respectively. It proves that solid-phase epitaxy is more suitable for the solar cell growth on the solid-phase crystallised silicon seed layer than vapour-phase epitaxy.
\end{abstract}

\section{Introduction}

Polycrystalline silicon (poly-Si) on glass thin-film solar cells combines advantages of crystalline silicon-wafer based technology with a low material usage and large area monolithic integration typical for thin-film technologies [1]. Poly-Si thinfilm can be made via epitaxy on a poly-Si seed layer. Alinduced crystallisation (AIC) is commonly used for poly-Si seed layer fabrication $[2,3]$. The grain size in the AIC seed layer is larger than $10 \mu \mathrm{m}$, but the large grain size does not lead to better solar cells. The use of Al for the AIC seed layer formation results in metal contamination leading to the electronically active intragrain defects. These intragrain defects extending to the epilayer are found to be strongly limiting the performance of the solar cell on the AIC seed layer [4]. Solid phase crystallization (SPC) of amorphous $\mathrm{Si}$ (a-Si) thin films is the simplest technique to obtain poly-Si at relatively low temperature $\left(600^{\circ} \mathrm{C}\right)$ and it is commonly used for poly-Si films solar cell fabrication $[5,6]$. It was reported that heavy P doping of about $1 \times 10^{20} \mathrm{~cm}^{-3}$ enhanced SPC and resulted in a high crystal and electronic quality poly-Si material $[7,8]$. Thus, a thin heavily P-doped SPC poly-Si film can be used as a seed layer for epitaxy and it can also act as the emitter in a respective solar cell.

Si epitaxy can be performed either directly from a vapour phase (VPE) or indirectly from a solid phase (SPE). For the VPE, the highest efficiency of $8 \%$ has been achieved on the AIC seed layer on an alumina substrate by thermal CVD at $1130^{\circ} \mathrm{C}$ [3]. However, VPE on the AIC seed layer on an inexpensive glass substrate results in a much poorer cell efficiency of $2.28 \%$ and a lower open circuit voltage of $346 \mathrm{mV}$ [9], since the use of the glass substrate limits processing temperatures to below $650^{\circ} \mathrm{C}$. In contrast to VPE methods, where Si atoms impinging the seed layer surface immediately form a crystalline film, the low-temperature $\left(<600^{\circ} \mathrm{C}\right) \mathrm{SPE}$ is an indirect method. It consists of two steps: (i) deposition of a-Si on the seed layer and (ii) solid-phase crystallisation of a-Si via epitaxy [10].

In this paper, the heavily phosphorous-doped SPC poly-Si seed layer was thickened by high rate electron beam (E-beam) evaporation. Because epitaxy on the SPC poly-Si seed layer can be performed by VPE or by SPE, a detailed comparison was made to identify which of two methods is suitable for obtaining higher quality poly-Si epilayers and thin-film solar 
cells. We also tried to find a relationship between intragrain defects in the seed layer and the epilayer on the one hand and the solar cell performance on the other hand.

\section{Experimental Procedure}

The seed layer precursor $\left(80 \mathrm{~nm} \mathrm{SiN}_{x} / 200 \mathrm{~nm}\right.$ a-Si with Pdoping concentration of about $3.3 \times 10^{20} \mathrm{~cm}^{-3}$ ) was deposited on a planar glass substrate (Schott Borofloat 33) by plasmaenhanced chemical vapour deposition. $\mathrm{SiN}_{x}$ acts as a barrier and antireflection layer. After deposition, all seed layer precursors were crystallised at $600^{\circ} \mathrm{C}$ in a tube furnace purged with nitrogen. After crystallisation, the seed layers were annealed at $950^{\circ} \mathrm{C}$ for 3 mins in a belt furnace heated by halogen lamps and purged with nitrogen to eliminate intragrain defects and activate the dopant [11].

The absorber and back surface field (BSF) were deposited on the seed layers by E-beam evaporation at $300 \mathrm{~nm} / \mathrm{min}$ with the doping profile as follows: $\sim 2 \mu \mathrm{m}, 2 \times 10^{16} \mathrm{~cm}^{-3}$ B-doped absorber and $\sim 100 \mathrm{~nm}, 2 \times 10^{19} \mathrm{~cm}^{-3} \mathrm{~B}$-doped BSF. VPE was conducted at the substrate temperature of about $630^{\circ} \mathrm{C}$. The aSi precursor for SPE was deposited at $250^{\circ} \mathrm{C}$ followed by SPE at $580^{\circ} \mathrm{C}$ in a nitrogen purged tube furnace.

Both the VPE and SPE grown samples received another annealing at $950^{\circ} \mathrm{C}$ for 3 mins to activate dopants and to reduce the defect density. Then, high-temperature hydrogenation treatment was applied to passivate the grain boundaries. Hydrogen passivation was performed in a cold-wall vacuum system with an inductively coupled remote plasma source at a temperature of $650^{\circ} \mathrm{C}$ for $20 \mathrm{~min}$, a plasma power of $3200 \mathrm{~W}$, hydrogen gas flow of $200 \mathrm{sccm}$, and argon gas flow of $60 \mathrm{sccm}$ [12].

The VPE and SPE grown samples were cut to size of $5 \mathrm{~cm} \times 5 \mathrm{~cm}$ and were metallised with interdigitated $\mathrm{Al}$ line contacts on the heavily doped layers, the emitter, and BSF. All cells were in the superstrate configuration, had an area of $2 \mathrm{~cm}^{2}$, and did not have any light trapping features. More details on the metallisation scheme used in this work are given in [13].

Intragrain defects in the epitaxial Si layers were analysed by cross-sectional transmission electron microscopy (TEM) (Philips CM200 with a field emission gun). To make defects visible, defect etching was performed using diluted Secco etch solution $\left(0.15 \mathrm{~mol} / \mathrm{L} \mathrm{K}_{2} \mathrm{Cr}_{2} \mathrm{O}_{7}: 50 \% \mathrm{HF}\right.$ : DI water, ratio $1: 2: 3$ ) on the sample surface [14]. The resulting etch pits were analysed by scanning electron microscopy (SEM) using an ultrahigh resolution field emission SEM (FEI Nova NanoSEM 230). Crystal structure quality of epitaxial Si films was evaluated from X-ray diffraction and Raman spectroscopy which were measured by the Philips X'pert Materials Research diffractometer operated at a voltage of $40 \mathrm{kV}$ and a current of $40 \mathrm{~mA}$, using $\mathrm{Cu} \mathrm{K} \alpha$ radiation $(\lambda$ $=1.54 \AA$ ) and PerkinElmer Raman station 400 with $785 \mathrm{~nm}$ laser, respectively. Reflectance spectra were measured by a Perkin Elmer UV-Vis spectrometer (Lambda 1050). External quantum efficiency (EQE) measurements were performed by a QEX10 spectral response system from PV Measurements, Inc. The illuminated current-voltage $(I-V)$ measurements were performed using an IV5 solar cell $I-V$ testing system from PV Measurement, Inc. (using a Keithley 2400 source meter) under illumination power of $100 \mathrm{~mW} \cdot \mathrm{cm}^{-2}$ by an AM $1.5 \mathrm{G}$ solar simulator (Oriel model 94023A).

\section{Results and Discussion}

3.1. Material Characterisations. In Figure 1(a), both VPE and SPE poly-Si thin films exhibit diffraction peaks at $2 \theta$ values of about $28.4^{\circ}, 47.3^{\circ}, 56.2^{\circ}$, and $69.1^{\circ}$ which correspond to the (111), (220), (311), and (400) orientations of c-Si, respectively. Interestingly, a shoulder peak $\left(111^{-}\right)$occurs at the low angle side of the main (111) reflection for the VPE sample. In order to get more detailed information on this shoulder peak, another scan was done with a longer step duration time from $3 \mathrm{~s}$ to $10 \mathrm{~s}$ to get more accurate data shown in Figure 1(b).

The new shoulder peak in the XRD spectra close to the classical Si-(111) orientation has been reported for plasmadeposited Si films [15], for a hydrogenated a-Si film after crystallisation by thermal annealing [16] and for poly-Si thin films prepared by low-pressure chemical vapour deposition (LPCVD) [17]. Hendriks et al. [17] suggested that this additional peak might be attributed to the presence of new polytypic $\mathrm{Si}$ in the poly-Si films. The polytypic $\mathrm{Si}$ is a hexagonal phase in which a large number of planar faults (stacking faults and microtwins) are formed. Recently, Cayron et al. [18] proved that this additional peak results from the double diffraction of overlapping $\Sigma 3$ microtwins and the stacking faults on the $\{111\}$ planes. Thus, the new shoulder peak $\left(111^{-}\right)$results from the high density of microtwins and stacking faults in poly-Si. This shoulder should not result from the defects in the RTA treated SPC seed layer because our previous results proved that RTA can effectively eliminate the intragrain defects in the SPC seed layer [11]. Therefore, the shoulder peak should be due to the intragrain defects in the epilayer rather than in the seed layer. The shoulder peak is only observed in the VPE sample and it is not found in the SPE sample. Therefore, it indicates that the planar defect density in the VPE poly-Si is much higher than that in the SPE one.

Due to the lower diffusivity of Si adatoms at low temperatures [19], formation of planar defects is an inherent property of low-temperature VPE growth. In particular, VPE on the (111)-oriented seed is more likely to form planar defects than on other orientations. It is reported that low temperature direct epitaxy on Si strongly depends on the seed layer or cSi substrate orientation by various direct epitaxial techniques such as molecular beam epitaxy (MBE) [19], ECR-CVD [20], and pulsed magnetron sputtering [21]. VPE on Si-(111) is different from that on $\mathrm{Si}-(100)$ due to the different bonding geometry of the Si atoms on the surface [20]. The formation energy of stacking faults is lower on $\mathrm{Si}$-(111) than on $\mathrm{Si}-(100)$ [22]. It was reported that stacking faults density in the epilayer is lower than $1 \times 10^{2} \mathrm{~cm}^{-2}$ on (100)-oriented c-Si. However, for VPE on (111)-oriented $\mathrm{c}-\mathrm{Si}$, the stacking faults density of $7 \times 10^{4} \mathrm{~cm}^{-2}$ is two orders higher than on $\mathrm{Si}-(100)$ [23]. Additionally, due to the lower diffusivity of Si adatoms at low temperatures, twin boundaries can form on $\{111\}$ plane 


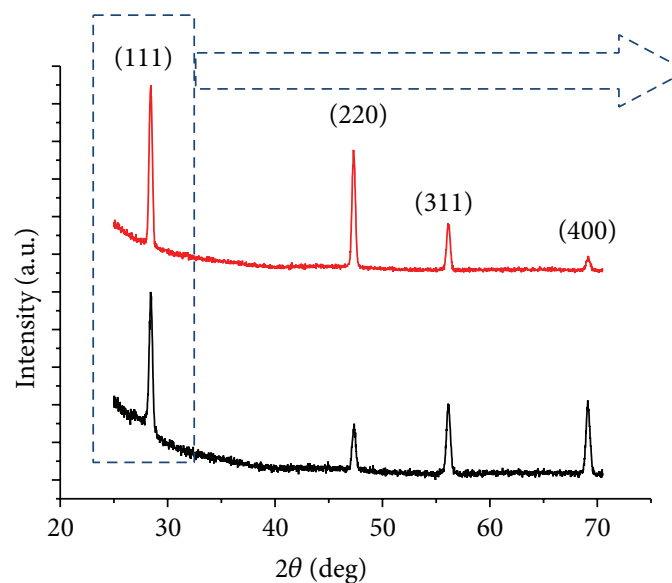

(a)

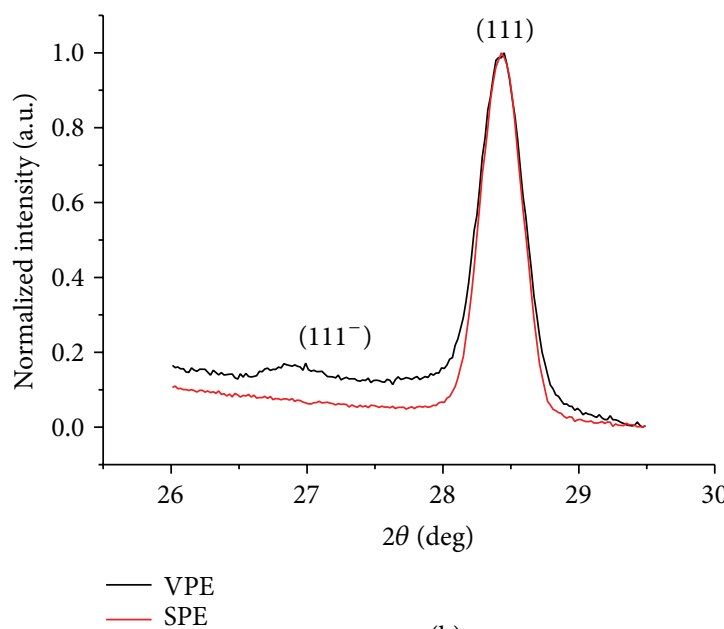

(b)

FIGURE 1: (a) The XRD pattern of the $2 \mu \mathrm{m}$ VPE and SPE grown poly-Si samples. (b) is the detailed XRD spectrum with two-theta range from $26^{\circ}$ to $29.5^{\circ}$.

TABLE 1: XRD peak FWHM and Raman peak FWHM of the VPE and SPE grown poly-Si epilayer.

\begin{tabular}{lccc}
\hline Method & Peak & VPE & SPE \\
\hline & $(111)$ & 0.35 & 0.33 \\
XRD peak FWHM $\left(^{\circ}\right)$ & $(220)$ & 0.36 & 0.33 \\
& $(311)$ & 0.38 & 0.35 \\
& $(400)$ & 0.36 & 0.34 \\
\hline Raman peak FWHM $\left(\mathrm{cm}^{-1}\right)$ & & $6.03 \pm 0.10$ & $5.65 \pm 0.05$
\end{tabular}

as they require relatively low energy [19]. Consequently, the formation of stacking faults and twins during epitaxial growth is much more likely on the Si-(111) surface than on the Si-(100) surface. That is why the shoulder peak only presents for Si-(111) orientation and is not found for Si-(100) orientation or some other Si orientations.

It is well known that the broadening of the FWHM of the Raman peak occurs due to a decrease in the phonon lifetimes which is mainly a result of defects acting as anharmonic perturbations. Therefore, Raman peak FWHM can be used to qualitatively estimate defect densities [24]. FWHM of the Raman peak is averaged over nine different positions taken over the area of $25 \times 25 \mu \mathrm{m}^{2}$.

Planar defects can also cause broadening of the Bragg diffraction peaks [17]. XRD measurement with a larger detection area $\left(12 \times 2 \mathrm{~mm}^{2}\right)$ determines the spatially averaged crystal quality of Si films which is complementary to Raman measurements. FWHM of XRD (111), (220), (311), and (400) orientations and FWHM of the Raman peak for the VPE and SPE poly-Si films are shown in Table 1. FWHM of XRD and Raman peaks for the VPE sample are larger than those for the SPE sample. Therefore, both XRD and Raman reveal that the SPE film has better crystallinity than the VPE film.

Cross-sectional TEM (XTEM) images are taken to check whether epitaxy has taken place and to investigate intragrain defects in the epilayers. Figures 2(a) and 2(b) are bright-field and dark-field images of the VPE sample on the P-doped SPC seed layer, respectively. Figures 2(c) and 2(d) are bright-field and dark-field images of the SPE sample on the P-doped SPC seed layer, respectively. It can be seen that grain boundaries in the SPC poly-Si seed layer propagate into the VPE and SPE layers in Figures 2(a) and 2(c). Figures 2(e) and 2(g) are the selective area electron diffraction (SAED) pattern of the circled regions " $A$ " and " $C$ " in Figures 2(b) and 2(d), respectively. The regions " $\mathrm{A}$ " and " $\mathrm{C}$ " are the interface between the seed layer and the epilayer. These two SAED patterns suggest that the orientation of the seed layers and VPE or SPE layers is the same. Therefore, Figure 2 confirms that a transfer of crystallographic information has taken place from the SPC seed layer into the VPE and SPE grown epilayers.

In Figure 2(b), the region "B" in the VPE layer has a large density of parallel lines. In Figure 2(f), the periodic extra spots in the reciprocal lattice are due to twins on $\{111\}$ plains so the parallel lines in Figure 2(b) are twins growing along [112] direction in the VPE grown epilayer. Satellite spots with forbidden indices 1/3(111) and 2/3(111) are Moiré patterns due to double diffraction of the electron beam by superimposing microtwins. It reveals a three-dimensional distribution of microtwins inside the grains [25]. This result supports the finding of the shoulder peak in the XRD spectrum which also results from the high density of planar defects in the VPE layer.

No twins are observed in the SAED pattern of Figure 2(e) at the interface region between VPE layer and the seed layer (region "A"). As an inherent property of low-temperature epitaxy [20], formation of planar defects tends to increase surface roughness, form $\{111\}$ facets, twin boundaries [19], and dislocations, and finally cause crystal quality degradation $[26,27]$. Thus, as the VPE growth on the SPC seed layer carries on, the transition from a well-ordered material into a defect rich structure takes place.

The large density of microtwins grown along [112] direction is the first-order $\Sigma 3$ twins which are reported to 


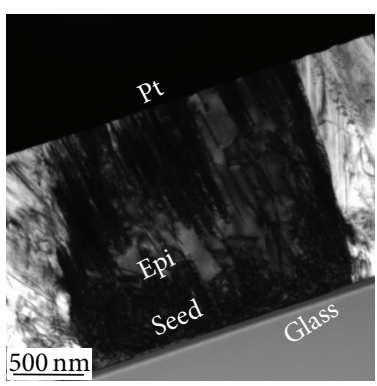

(a)

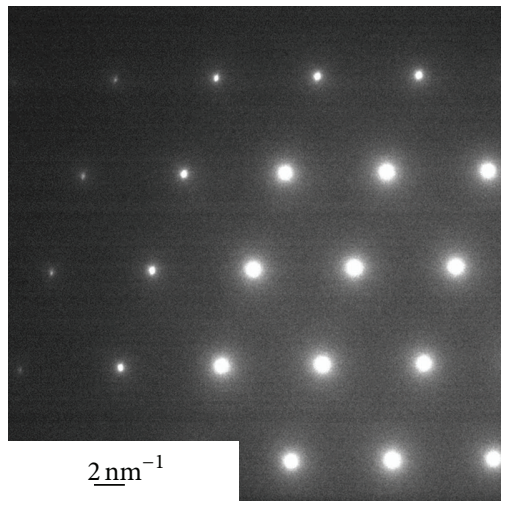

(e)

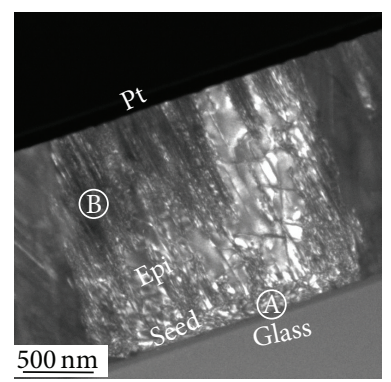

(b)

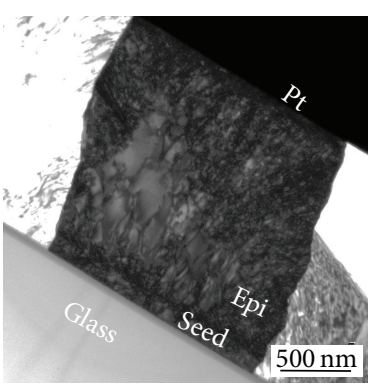

(c)

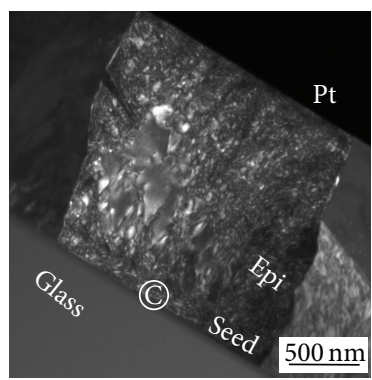

(d)

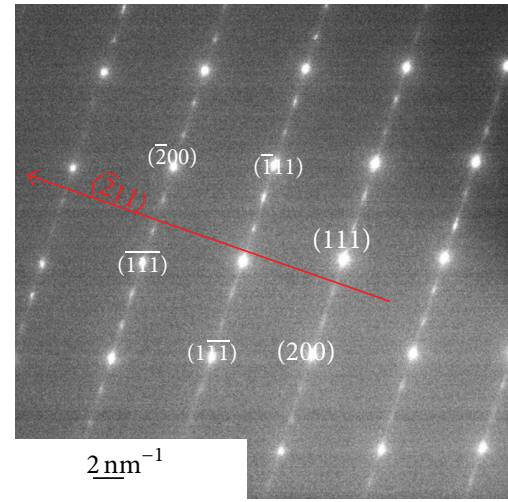

(f)

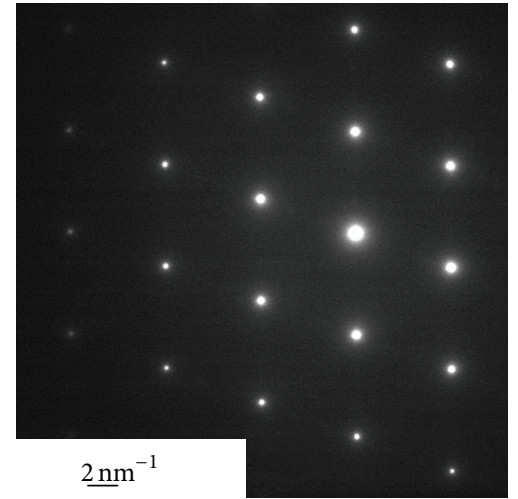

(g)

FIGURE 2: (a) and (b) XTEM bright- and dark-field images of the VPE grown sample on the SPC seed, respectively; (c) and (d) XTEM brightand dark-field images of the SPE grown sample on the SPC seed layer, respectively; (e), (f), and (g) SAED pattern of the circled areas "A" and "B" in (b) and "C" in (d), respectively.

be electrically inactive [28]. However, when they terminate inside grains, they can introduce electrically active higher order twins or dislocations [25] which are expected to act as recombination centres for free-charge carriers detrimental to the solar cell performance.

The planar defect density in the SPE sample is much lower than that in the VPE sample from comparison between Figures 2(b) and 2(d). It is consistent with the results from XRD and Raman spectra that the structural quality of the SPE layer is better than that of the VPE layer. Moreover, as the SPE on the SPC seed layer carries on, the quality of the SPE layer does not show degradation as for the VPE layer. Stradins et al. [29] reported that as SPE films grew thicker, the epilayer crystalline quality improved. They explained this phenomenon by attributing the interface defects to the initial defective epitaxial layer formed at the stages of a-Si film deposition.

The XTEM image also shows that the SPE film has a distinctive noncolumnar structure with nonvertical grain boundaries which is quite different from the VPE film that has a columnar structure with vertical grain boundaries. The columnar structure of the VPE grain could be related to the fact that E-beam evaporation is a highly directional deposition method [13] with a fast deposition rate at $300 \mathrm{~nm} / \mathrm{min}$. During VPE growth, the film deposition process and grain crystallization is completed simultaneously. At such high deposition rate, the evaporated $\mathrm{Si}$ atoms do not have enough time to move to some specific oriented seeds to get a faster epitaxial growth rate but stay at where they arrived at the seed layer surface. Therefore, the grain growth rate in the vertical direction of the glass substrate is determined by the deposition rate rather than on the seed layer orientation. Finally, a column-like grain structure is formed by VPE growth. However, it is possible that in some orientation the epitaxy is failed due to high defect density in the epilayer.

A plausible explanation for the noncolumnar structure is a grain orientation-dependent crystal growth rate [10]. It can be imagined that SPE growth takes place on two adjacent seed grains, one is (100) oriented and the other is (111) oriented. Due to the anisotropic growth rate, the SPE on the (100)oriented grain permits a faster growth in the direction to the surface. The grain also starts gradually growing laterally at the expense of the adjacent more slowly growing (111)-oriented grain. Grains preferentially oriented for fast growth expand at the expense of slowly growing grains. Therefore, the grain boundaries are not vertical to the seed layer and the film has noncolumn grain structure.

Intragrain defects are considered to be limiting the electronic quality of poly-Si films [30]. Secco etching can make the intragrain defects (dislocations, stacking faults, and twins) visible which facilitates the analysis of the crystal quality [14]. Crystallographic defects present in the epitaxial layers prepared by VPE and SPE on the SPC poly-Si seed layer were investigated by combining defect etching with SEM imaging. Note that the VPE sample is much more defective than the SPE sample, so different etching time was used. 
The VPE sample was etched for $15 \mathrm{~s}$ while the SPE sample was etched for $60 \mathrm{~s}$. Figures 3(a) and 3(b) are two samescale SEM images of the defect-etched VPE and SPE samples, respectively. In both images, the defect-etched marks consist mostly of points and lines, attributed to dislocations and planar defects (stacking faults and twins), respectively [4, 31].

Although it is hard to identify grain boundaries in the VPE sample, it is relatively easy to distinguish a good region (region A) with lower density of planar defects from a planar defect-rich region (region B). Defect density in the VPE layer depends on the seed orientation. On the epilayer grown on (100)-oriented Si wafers by E-beam evaporation, no etch pits could be detected after Secco etching. In contrast, the defect densities in the epilayer grown on (110)- and (111)oriented Si wafers were determined to be $\sim 8 \times 10^{6} \mathrm{~cm}^{-2}$ and $\sim 2 \times 10^{8} \mathrm{~cm}^{-2}$, respectively [32]. As we also discussed above, the formation of stacking faults and twins during VPE is much more likely on the Si-(111) surface than on the Si-(100) surface. Thus, the seed layer orientation strongly influences the structural quality of the VPE layer. A SPC poly-Si film is a randomly oriented material [33] so epitaxial growth on the SPC seed layer results in a nonuniform defect density distribution in the epilayer.

The planar defects are still evident in the SPE sample and these planar defects (twins) are marked with arrows in Figure 3(b). The formation of these twins can be explained as follows.

Formation of a $\{111\}$ twin boundary can enhance the SPE rate, especially on (111)-oriented seed. The SPE rate is the slowest on the (111)-oriented seed since more amorphous atoms are needed for completion of a sixfold ring of atoms at the perfect crystalline interface. Once stacking faults or twins are formed on inclined $\{111\}$ planes, the SPE growth rate on the (111)-oriented seed is greatly accelerated due to reduction of the required coordination number of surrounding atoms [34]. Moreover, most of the microtwins are absorbed by the larger twins during the RTA process after epitaxy [25]. Therefore, these larger twins are still shown in Figure 3(b). However, they completely penetrate the whole crystal without introducing other electrically active defects [28] so they should not harm the electronic quality of poly-Si thin films.

Comparing Figures 3(a) and 3(b), planar defect density in the SPE sample is much lower than that in the VPE sample. It indicates that the structural quality of the SPE sample is better than for the VPE sample as it was found from the other data discussed above. Moreover, in the VPE sample, some of the regions have a much higher density of planar defects than the other regions which results from the randomly oriented SPC seed layer. In contrast, the planar defects are uniformly distributed in the SPE sample. Thus, the seed layer orientation does not seem to significantly influence the defect density in the SPE epilayer which is not the same as for the VPE epilayer.

3.2. Solar Cell Performance. The performance parameters for VPE and SPE solar cells before and after metallisation are shown in Table 2. The $1-$ Sun $-V_{\text {oc }}$ before metallisation was averaged over five different positions over a $5 \times 5 \mathrm{~cm}^{2}$ sample area. After metallisation, the $V_{\text {oc }}$ of VPE and SPE samples increases from $385 \mathrm{mV}$ to $399 \mathrm{mV}$ and from $442 \mathrm{mV}$
TABLE 2: Performance parameters for the VPE and SPE grown solar cells.

\begin{tabular}{lccc}
\hline Cell & & VPE & SPE \\
\hline Suns- $V_{\text {oc }}(\mathrm{mV})$ & Before metal. & $385 \pm 6$ & $442 \pm 3$ \\
& After metal. & 399 & 460 \\
EQE & $J_{\text {sc }}\left(\mathrm{mA} / \mathrm{cm}^{2}\right)$ & 7.75 & 10.15 \\
\hline
\end{tabular}

TABLE 3: The fitted results for the EQE and reflectance spectrum by PC1D simulation.

\begin{tabular}{lcccc}
\hline Sample & $t_{\mathrm{emi}}(\mu \mathrm{m})$ & $L_{\mathrm{emi}}(\mu \mathrm{m})$ & $t_{\mathrm{abs}}(\mu \mathrm{m})$ & $L_{\mathrm{abs}}(\mu \mathrm{m})$ \\
\hline SPE & 0.6 & 0.37 & 1.5 & 2.67 \\
VPE & 0.8 & 0.35 & 1.3 & 1.42 \\
\hline
\end{tabular}

to $460 \mathrm{mV}$, respectively. The $V_{\text {oc }}$ after metallisation is higher than the $V_{\text {oc }}$ before metallisation due to the better contact to BSF and the emitter.

The minority carrier life time is reflected in the modest $V_{\text {oc }}$ of poly-Si thin-film solar cells as shown in the equation below [35]:

$$
V_{\mathrm{oc}}=E_{g}-\frac{k T}{q} \ln \left(\frac{q N_{c} N_{v} W}{J_{\mathrm{sc}} n_{0} \tau}\right),
$$

where $E_{g}$ is the band gap, $k T$ is the thermal energy, $q$ is the elementary charge, $N_{c}$ and $N_{v}$ are the effective density of states of the conduction and valence bands, respectively, $n_{0}$ is the equilibrium majority carrier concentration in the base, $J_{\mathrm{sc}}$ is the short-circuit current density, $W$ is the width of the absorber, and $\tau$ is the low injection level minority carrier lifetime. Thus, analysis of $V_{\text {oc }}$ can reveal the trend in the minority carrier life time in the material [35]. In Table 2, $V_{\mathrm{oc}}$ of the SPE sample is about $60 \mathrm{mV}$ higher than that of the VPE sample indicating that the material quality of the SPE sample is much better than that of the VPE sample.

As seen in Figure 4, EQE of the SPE cell is higher than $\mathrm{EQE}$ of the VPE cell over the whole wavelength range. Consequently, after integrating the EQE over the AM1.5 spectrum, $J_{\mathrm{sc}}$ of the SPE cell $\left(10.15 \mathrm{~mA} / \mathrm{cm}^{2}\right)$ is also much higher than $J_{\mathrm{sc}}$ of the VPE cell $\left(7.75 \mathrm{~mA} / \mathrm{cm}^{2}\right)$ as shown in Table 2.

In order to better understand the differences in the spectral response, PC1D modelling of the EQE and the reflectance was carried out. The following parameters were used: BSF thickness $100 \mathrm{~nm}$, BSF doping $4 \times 10^{19} \mathrm{~cm}^{-3}$, and refractive indices $n_{\text {air }}=1$ and $n_{\text {glass }}=1.48$. The cell thickness was fixed at $2.1 \mu \mathrm{m}$ as it was measured from XTEM. The rear surface recombination velocity was found to have a negligible effect on the modelling results, indicating that surface passivation was not important for the investigated cell performance [36]. The parameters listed in Table 3 were varied until the experimental data and the model curves were in satisfactory agreement.

A close match was achieved between the experimental and simulated data for the SPE sample, as shown in Figure 4. Although over the most of the wavelength range the fitted curve closely matches the experimental data, some minor deviations still exist. The deviation in the reflection curves at 


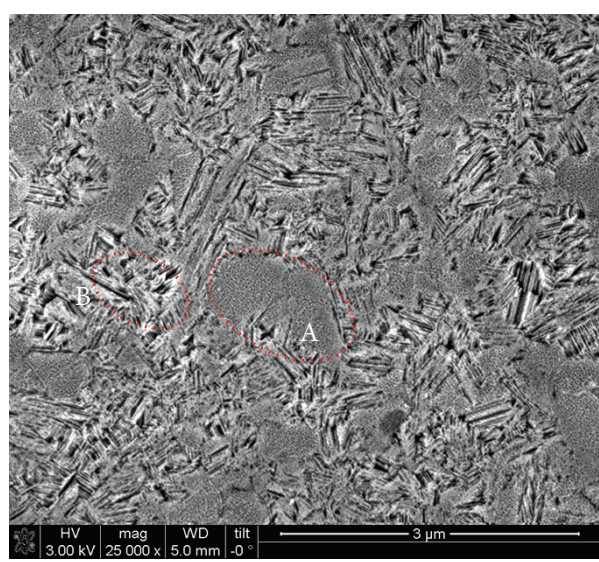

(a)

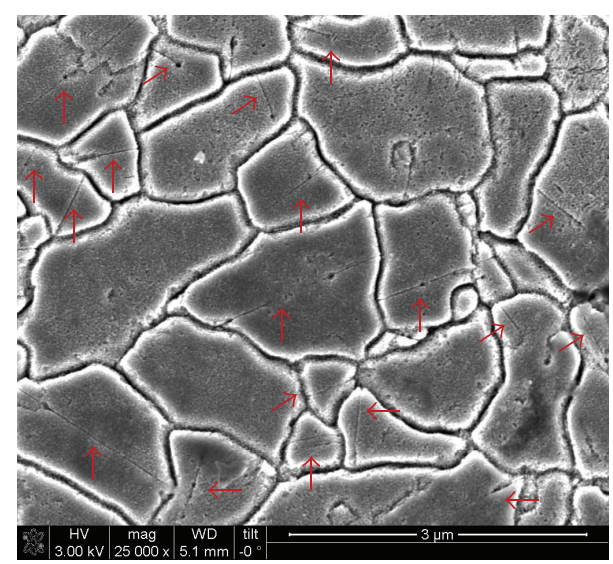

(b)

FIGURE 3: SEM image of Secco etched VPE and SPE thickened poly-Si films. (a) VPE sample after $15 \mathrm{~s}$ Secco etching and (b) SPE sample after Secco etching for $60 \mathrm{~s}$ (planar defects are noted by arrows in (b)).

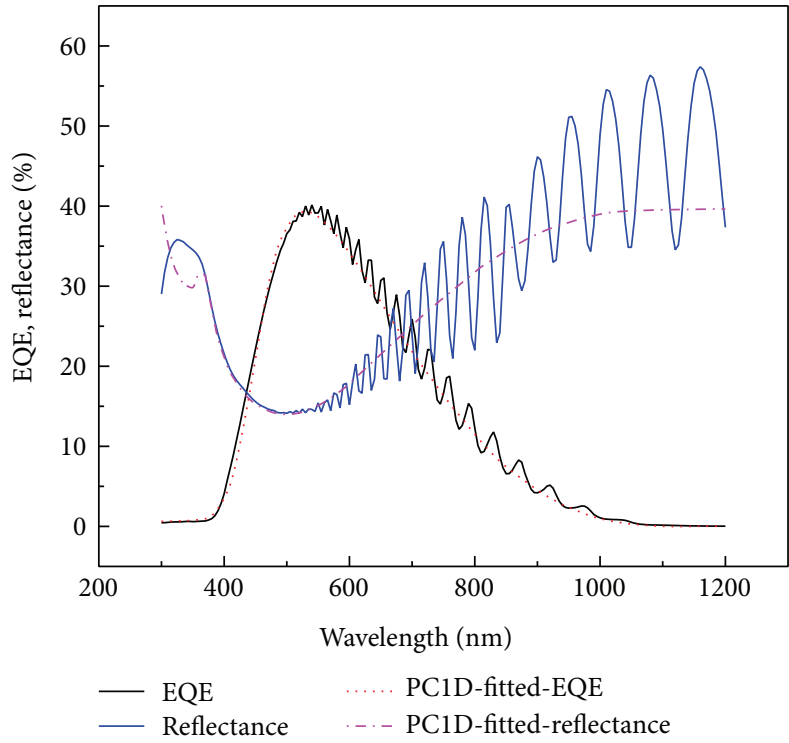

(a)

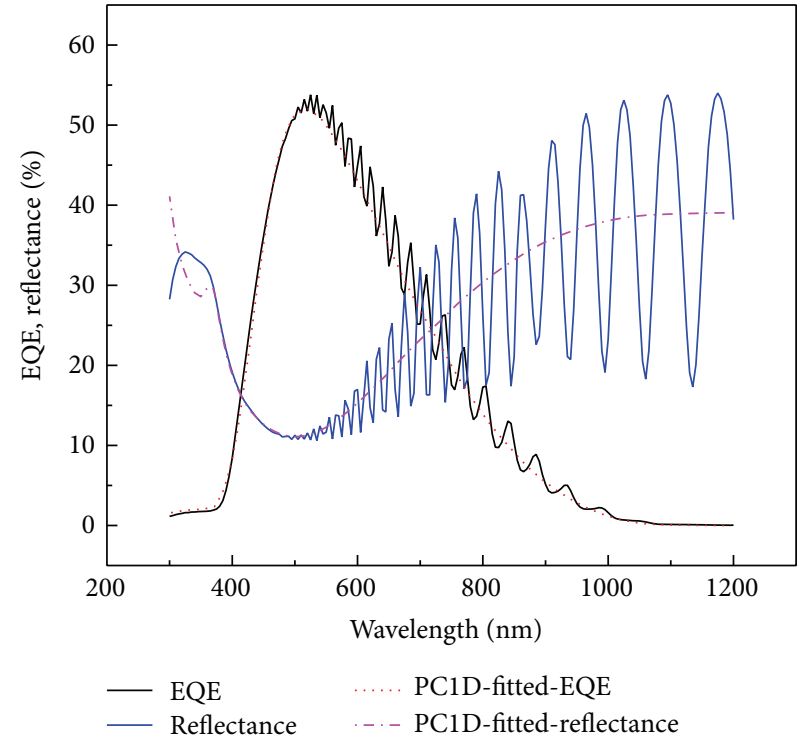

(b)

FIGURE 4: Measured EQE (black solid line), PC1D modelled EQE (red dot line), measured reflectance (blue solid line), and PC1D modelled reflectance (pink dashed dot line) of (a) the VPE and (b) the SPE cells.

wavelengths shorter than $400 \mathrm{~nm}$ is due to the limitation of PC1D that assumes a single value for the refractive index and zero absorption for the $\mathrm{SiN}_{x}$ layer. Also, due to the fact that PC1D only models the noncoherent optical properties and does not include thin-film interference effects, the measured reflectance results are slightly lower than the modelled ones in the long wavelength region and no interference fringes are seen in the modelled reflectance curves [36].

As shown in Table 3, the PC1D fitted emitter thickness $\left(t_{\text {emi }}\right)$ of VPE cell $(0.8 \mu \mathrm{m})$ is larger than that of the SPE cell $(0.6 \mu \mathrm{m})$ while the minority carrier diffusion length in the emitter $\left(L_{\mathrm{emi}}\right)$ is almost the same. The emitter of the VPE cell is deeper than that of the SPE cell. This finding is also supported by the fact that EQE peak positions of the
VPE cell move to the longer wavelengths compared with the SPE cell. During RTA process after epitaxial growth, dopants smear from the heavily P-doped emitter (seed layer) into the absorber [37]. Thus, the width of the absorber region is reduced while the emitter becomes wider than prior to RTA. $\mathrm{P}$ diffusion within the poly-Si layer is strongly affected by the grain microstructure due to the segregation of $\mathrm{P}$ at the grain boundaries where the diffusivity of $\mathrm{P}$ is 2 to 3 orders of magnitude larger than that in the bulk [38]. Therefore, due to $\mathrm{P}$ segregation at grain boundaries, it is possible that $\mathrm{P}$ atoms form spikes from the seed layer into the absorber. Deeper spikes are easier to form in the VPE cells with vertical grain boundaries as shown in XTEM images than in the SPE cells with the noncolumnar structure. Thus, the $p-n$ junction in 
the VPE cells can appear deeper than in the SPE cells. Thus, despite the fact that the P-doped poly-Si seed layer for epitaxy is completely the same, the modelled emitter thickness of the VPE cells is larger than that of the PE cells due to P atom diffusion along the vertical grain boundaries.

The PC1D fitted minority carrier diffusion length in the absorber $\left(L_{\text {abs }}\right)$ of SPE cell $(2.67 \mu \mathrm{m})$ is much larger than that of the VPE cell $(1.42 \mu \mathrm{m})$. The $L_{\mathrm{abs}}$ and the thickness of the absorber $\left(t_{\mathrm{abs}}\right)$ are two major parameters to determine the collection probability of the light-generated carriers. If $L_{\text {abs }}$ is smaller than $t_{\mathrm{abs}}$, light-generated carriers recombine in the absorber before collection by the junction. From the PC1D simulation results, $L_{\mathrm{abs}}$ is approximately equal to $t_{\mathrm{abs}}$ in the VPE cell but $L_{\text {abs }}$ of the SPE cell is much larger than its absorber thickness. Minority carriers in the SPE cell are more likely to be collected before recombination than in the VPE cell.

In the VPE growth, $L_{\mathrm{abs}}$ is reported to be related to the seed layer orientation. For the VPE on (100)-oriented c-Si wafer, the solar cell has $L_{\text {abs }}$ of $7 \mu \mathrm{m}$. However, for the VPE on (111)-oriented c-Si wafer, the cell has a lower bound of $3 \mu \mathrm{m}$ for $L_{\text {abs }}$ [39]. Lower value of $L_{\text {abs }}$ on (111)-oriented seed should result from the higher planar defects density in the epilayer on the (111)-oriented seed. However, the SPC seed layer is randomly oriented [31]; (111)-oriented seeds are unavoidable. Therefore, $L_{\text {abs }}$ of the whole VPE cell is limited by the part of epitaxy on (111)-oriented seed with much lower $L_{\text {abs }}$.

The illuminated $I-V$ characteristics of the VPE and SPE solar cells are shown in Figure 5. The SPE cell has higher $V_{\text {oc }}$ than the VPE cell. Although this trend is consistent with the $V_{\text {oc }}$ result obtained from Suns- $V_{\text {oc }}$, the value of $V_{\text {oc }}$ obtained by the $I-V$ curve is smaller than that obtained from Suns- $V_{\mathrm{oc}}$. The $J_{\mathrm{sc}}$ obtained from light $I-V$ curve is quite close to the result from the spectral response. Light $I-V$ measurements are strongly affected by the metallisation scheme in use [36] because the device final performance is not only related to the material quality but also determined by the metallization process. Nevertheless, the trend obtained from light $I-V$ characteristics is consistent with the Suns$V_{\mathrm{oc}}$ and EQE measurements in which the SPE poly-Si cell exhibits much higher $V_{\mathrm{oc}}, J_{\mathrm{sc}}$, and the photovoltaic conversion efficiency than the VPE cell. Without texture and back surface reflector, the SPE cell has the efficiency of $2.75 \%$ while the VPE cell efficiency is only $1.69 \%$.

The differences in the solar cell performance can be understood from the material quality differences between the VPE and SPE layer. From the material characterisation results, VPE on the SPC poly-Si seed layer leads to much higher planar defect (microtwins or stacking faults) density in the epilayer than SPE on the same seed layer. Microtwins introduce tail states into the energy band gap and the tail states lead to an inferior electric quality [40]. Moreover, microtwins can introduce other electronically active defects such as dislocations and high order twins $[25,35]$ which are detrimental to the solar cell performance. Therefore, the high planar defect density in the VPE layer limits the respective solar cell performance. The SPE cell does not have such a high planar defect density and thus achieves better performance than the VPE cell.

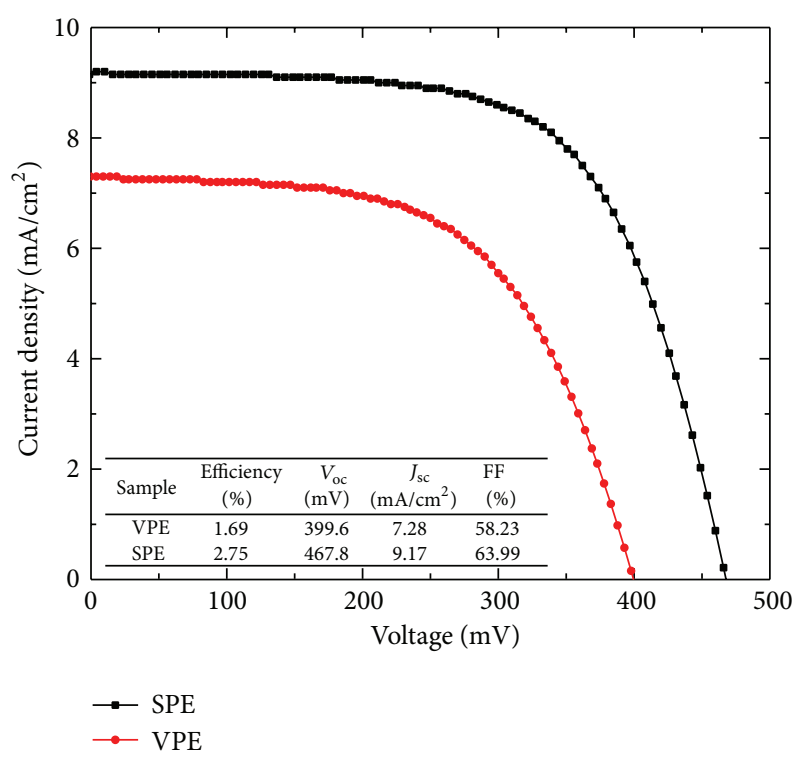

FIGURE 5: Light $I-V$ curves of the VPE and SPE grown poly-Si thinfilm solar cells on SPC seed layer.

\section{Conclusion}

Poly-Si thin-film solar cells are fabricated on glass superstrates via VPE or SPE growth on the heavily phosphorousdoped SPC poly-Si seed layer. A transfer of crystallographic information from the seed layer into the epilayer takes place during both processes. A much higher density of intragrain defects is observed in the VPE layer than in the SPE layer on the same SPC poly-Si seed layer. These intragrain defects in the VPE cell are strongly detrimental to the device performance. Consequently, the VPE solar cell has inferior material quality, much shorter minority diffusion length, and poorer performance than the SPE cell. Therefore, SPE is shown to be more suitable than VPE for solar cell fabrication by epitaxy on the SPC poly-Si seed layer.

\section{Conflict of Interests}

The authors declare that there is no conflict of interests regarding the publication of this paper.

\section{Acknowledgments}

This Program has been supported by the Australian Government through the Australian Renewable Energy Agency (ARENA) and Australian Research Council (ARC). Responsibility for the views, information, or advice expressed herein is not accepted by the Australian Government. The authors acknowledge the facilities and the scientific and technical assistance of the Australian Microscopy \& Microanalysis Research Facility at the Electron Microscope Unit, The University of New South Wales (UNSW). Wei Li would like to appreciate the Ph.D. scholarship from UNSW and China Scholarship Council (CSC). 


\section{References}

[1] M. A. Green, "Polycrystalline silicon on glass for thin-film solar cells," Applied Physics A: Materials Science \& Processing, vol. 96, no. 1, pp. 153-159, 2009.

[2] O. Nast, S. Brehme, S. Pritchard, A. G. Aberle, and S. R. Wenham, "Aluminium-induced crystallisation of silicon on glass for thin-film solar cells," Solar Energy Materials and Solar Cells, vol. 65, no. 1, pp. 385-392, 2001.

[3] I. Gordon, L. Carnel, D. van Gestel, G. Beaucarne, and J. Poortmans, " $8 \%$ efficient thin-film polycrystalline-silicon solar cells based on aluminum-induced crystallization and thermal CVD," Progress in Photovoltaics: Research and Applications, vol. 15, no. 7, pp. 575-586, 2007.

[4] D. van Gestel, I. Gordon, H. Bender et al., "Intragrain defects in polycrystalline silicon layers grown by aluminum-induced crystallization and epitaxy for thin-film solar cells," Journal of Applied Physics, vol. 105, no. 11, Article ID 114507, 2009.

[5] T. Matsuyama, N. Terada, T. Baba et al., "High-quality polycrystalline silicon thin film prepared by a solid phase crystallization method," Journal of Non-Crystalline Solids, vol. 198-200, no. 2, pp. 940-944, 1996.

[6] M. Keevers, U. Schubert, R. Evans, R. J. Egan, and M. A. Green, "10\% efficient CSG minimodules", in Proceedings of the 22nd European Photovoltaic Solar Energy Conference and Exhibition (EUPVSEC '07), p. 1783, Milan, Italy, 2007.

[7] S. He and A. B. Sproul, "Optical properties of evaporated poly-Si thin-films on glass," Thin Solid Films, vol. 519, no. 1, pp. 351-356, 2010.

[8] T. Matsuyama, M. Tanaka, S. Tsuda, S. Nakano, and Y. Kuwano, "Improvement of n-type poly-Si film properties by solid phase crystallization method," Japanese Journal of Applied Physics, vol. 32, pp. 3720-3728, 1993.

[9] S. Gall, C. Becker, E. Conrad et al., "Polycrystalline silicon thinfilm solar cells on glass," Solar Energy Materials and Solar Cells, vol. 93, no. 6-7, pp. 1004-1008, 2009.

[10] P. I. Widenborg, A. Straub, and A. G. Aberle, "Epitaxial thickening of AIC poly-Si seed layers on glass by solid phase epitaxy," Journal of Crystal Growth, vol. 276, no. 1-2, pp. 19-28, 2005.

[11] W. Li, S. Varlamov, J. Dore, and M. Green, "Defect annealing in ultra-thin polycrystalline silicon films on glass: rapid thermal versus laser processing," Materials Letters, vol. 107, pp. 1-4, 2013.

[12] P. I. Widenborg and A. G. Aberle, "Polycrystalline silicon thinfilm solar cells on AIT-textured glass superstrates," Advances in OptoElectronics, vol. 2007, Article ID 24584, 7 pages, 2007.

[13] O. Kunz, J. Wong, J. Janssens, J. Bauer, O. Breitenstein, and A. G. Aberle, "Shunting problems due to sub-micron pinholes in evaporated solid-phase crystallised poly-Si thin-film solar cells on glass," Progress in Photovoltaics: Research and Applications, vol. 17, no. 1, pp. 35-46, 2009.

[14] F. S. d'Aragona, "Dislocation etch for (100) planes in silicon," Journal of the Electrochemical Society, vol. 119, no. 7, pp. 948-951, 1972.

[15] G. Bruno, P. Capezzuto, F. Cramarossa, and R. D’Agostino, "R.f. plasma deposition of amorphous silicon films from $\mathrm{SiCl}_{4}-\mathrm{H}_{2}$," Thin Solid Films, vol. 67, no. 1, pp. 103-108, 1980.

[16] R. Bilenchi, I. Gianinoni, and M. Musci, "Hydrogenated amorphous silicon growth by $\mathrm{CO}_{2}$ laser photodissociation of silane," Journal of Applied Physics, vol. 53, no. 9, pp. 6479-6481, 1982.
[17] M. Hendriks, S. Radelaar, A. M. Beers, and J. Bloem, "Additional $\mathrm{X}$-ray and electron diffraction peaks of polycrystalline silicon films," Thin Solid Films, vol. 113, no. 1, pp. 59-72, 1984.

[18] C. Cayron, M. Den Hertog, L. Latu-Romain et al., "Odd electron diffraction patterns in silicon nanowires and silicon thin films explained by microtwins and nanotwins," Journal of Applied Crystallography, vol. 42, no. 2, pp. 242-252, 2009.

[19] M. V. Ramana Murty and H. A. Atwater, "Crystalstateamorphous-state transition in low-temperature silicon homoepitaxy," Physical Review B, vol. 49, no. 12, pp. 8483-8486, 1994.

[20] J. Platen, B. Selle, I. Sieber, S. Brehme, U. Zeimer, and W. Fuhs, "Low-temperature epitaxial growth of Si by electron cyclotron resonance chemical vapor deposition," Thin Solid Films, vol.381, no. 1, pp. 22-30, 2001.

[21] F. Fenske, S. Schulze, M. Hietschold, and M. Schmidbauer, "Structural investigations of homoepitaxial Si films grown at low temperature by pulsed magnetron sputtering on $\mathrm{Si}(111)$ substrates," Thin Solid Films, vol. 516, no. 15, pp. 4777-4783, 2008.

[22] J. Schwarzkopf, B. Selle, M. Schmidbauer, and W. Fuhs, "Lowtemperature epitaxial growth of B doped $\mathrm{Si}$ films on $\mathrm{Si}(100)$ and Si(111)," Thin Solid Films, vol. 427, no. 1-2, pp. 274-278, 2003.

[23] T. A. Wagner, L. Oberbeck, and R. B. Bergmann, "Low temperature epitaxial silicon films deposited by ion-assisted deposition," Materials Science and Engineering B: Solid-State Materials for Advanced Technology, vol. 89, no. 1-3, pp. 319-322, 2002.

[24] G. Sarau, S. Christiansen, R. Lewandowska, and B. Roussel, "Future of Raman in PV development," in Proceedings of the 35th IEEE Photovoltaic Specialists Conference (PVSC '10), pp. 17701775, IEEE, Honolulu, Hawaii, USA, June 2010.

[25] L. Haji, P. Joubert, J. Stoemenos, and N. A. Economou, "Mode of growth and microstructure of polycrystalline silicon obtained by solid-phase crystallization of an amorphous silicon film," Journal of Applied Physics, vol. 75, no. 8, pp. 3944-3952, 1994.

[26] D. J. Eaglesham, "Semiconductor molecular-beam epitaxy at low temperatures," Journal of Applied Physics, vol. 77, no. 8, pp. 3597-3617, 1995.

[27] Q. Wang, C. W. Teplin, P. Stradins, B. To, K. M. Jones, and H. M. Branz, "Significant improvement in silicon chemical vapor deposition epitaxy above the surface dehydrogenation temperature," Journal of Applied Physics, vol. 100, no. 9, Article ID 093520, 2006.

[28] J. Chen, D. Yang, Z. Xi, and T. Sekiguchi, "Recombination activity of $\Sigma 3$ boundaries in boron-doped multicrystalline silicon: influence of iron contamination," Journal of Applied Physics, vol. 97, no. 3, Article ID 033701, 2005.

[29] P. Stradins, Y. Yan, R. Reedy et al., "Physics of solid-phase epitaxy of hydrogenated amorphous silicon for thin film $\mathrm{Si}$ photovoltaics," in Amorphous and Polycrystalline Thin-Film Silicon Science and Technology 2006, S. Wagner, V. Chu, H. A. Atwater, K. Yamamoto, and H. W. Zan, Eds., vol. 910, p. 365, Materials Research Society, Warrendale, Pa, USA, 2007.

[30] D. V. Gestel, M. J. Romero, I. Gordon et al., "Electrical activity of intragrain defects in polycrystalline silicon layers obtained by aluminum-induced crystallization and epitaxy," Applied Physics Letters, vol. 90, no. 9, Article ID 092103, 2007.

[31] B. Rau, K. Petter, I. Sieber et al., "Extended defects in Si films epitaxially grown by low-temperature ECRCVD," Journal of Crystal Growth, vol. 287, no. 2, pp. 433-437, 2006. 
[32] B. Gorka, P. Dogan, I. Sieber, F. Fenske, and S. Gall, "Lowtemperature epitaxy of silicon by electron beam evaporation," Thin Solid Films, vol. 515, no. 19, pp. 7643-7646, 2007.

[33] F. Liu, M. J. Romero, K. M. Jones et al., "Solid-phase crystallization of evaporated silicon thin films on glass for photovoltaics: a combined SEM and TEM study," in Thin Film Solar Technology, vol. 7409 of Proceedings of SPIE, San Diego, Calif, USA, August 2009, 740906.

[34] R. Drosd and J. Washburn, "Some observations on the amorphous to crystalline transformation in silicon," Journal of Applied Physics, vol. 53, no. 1, pp. 397-403, 1982.

[35] J. Wong, J. L. Huang, B. Eggleston et al., "Lifetime limiting recombination pathway in thin-film polycrystalline silicon on glass solar cells," Journal of Applied Physics, vol. 107, no. 12, Article ID 123705, 2010.

[36] Z. Ouyang, O. Kunz, A. B. Sproul, and S. Varlamov, "Influence of the absorber doping for p-type polycrystalline silicon thinfilm solar cells on glass prepared by electron beam evaporation and solid-phase crystallization," Journal of Applied Physics, vol. 109, no. 5, Article ID 054510, 2011.

[37] M. L. Terry, A. Straub, D. Inns, D. Song, and A. G. Aberle, "Large open-circuit voltage improvement by rapid thermal annealing of evaporated solid-phase-crystallized thin-film silicon solar cells on glass," Applied Physics Letters, vol. 86, no. 17, Article ID 172108, 2005.

[38] S. Batra, K. H. Park, S. K. Banerjee et al., "Effect of grain microstructure on $\mathrm{P}$ diffusion in polycrystalline-on-single crystal silicon systems," Journal of Electronic Materials, vol. 21, no. 2, pp. 227-231, 1992.

[39] D. H. Neuhaus, N.-P. Harder, S. Oelting et al., "Dependence of the recombination in thin-film Si solar cells grown by ionassisted deposition on the crystallographic orientation of the substrate," Solar Energy Materials and Solar Cells, vol. 74, no. 1-4, pp. 225-232, 2002.

[40] M. Miyasaka and J. Stoemenos, "Excimer laser annealing of amorphous and solid-phase-crystallized silicon films," Journal of Applied Physics, vol. 86, no. 10, pp. 5556-5565, 1999. 

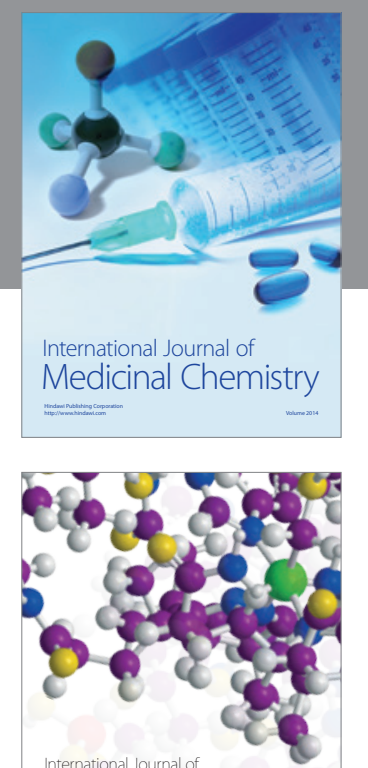

\section{Carbohydrate} Chemistry

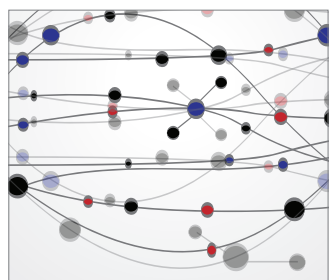

The Scientific World Journal
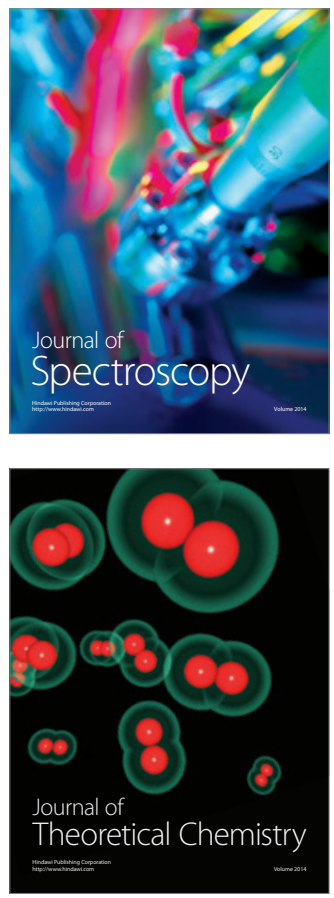
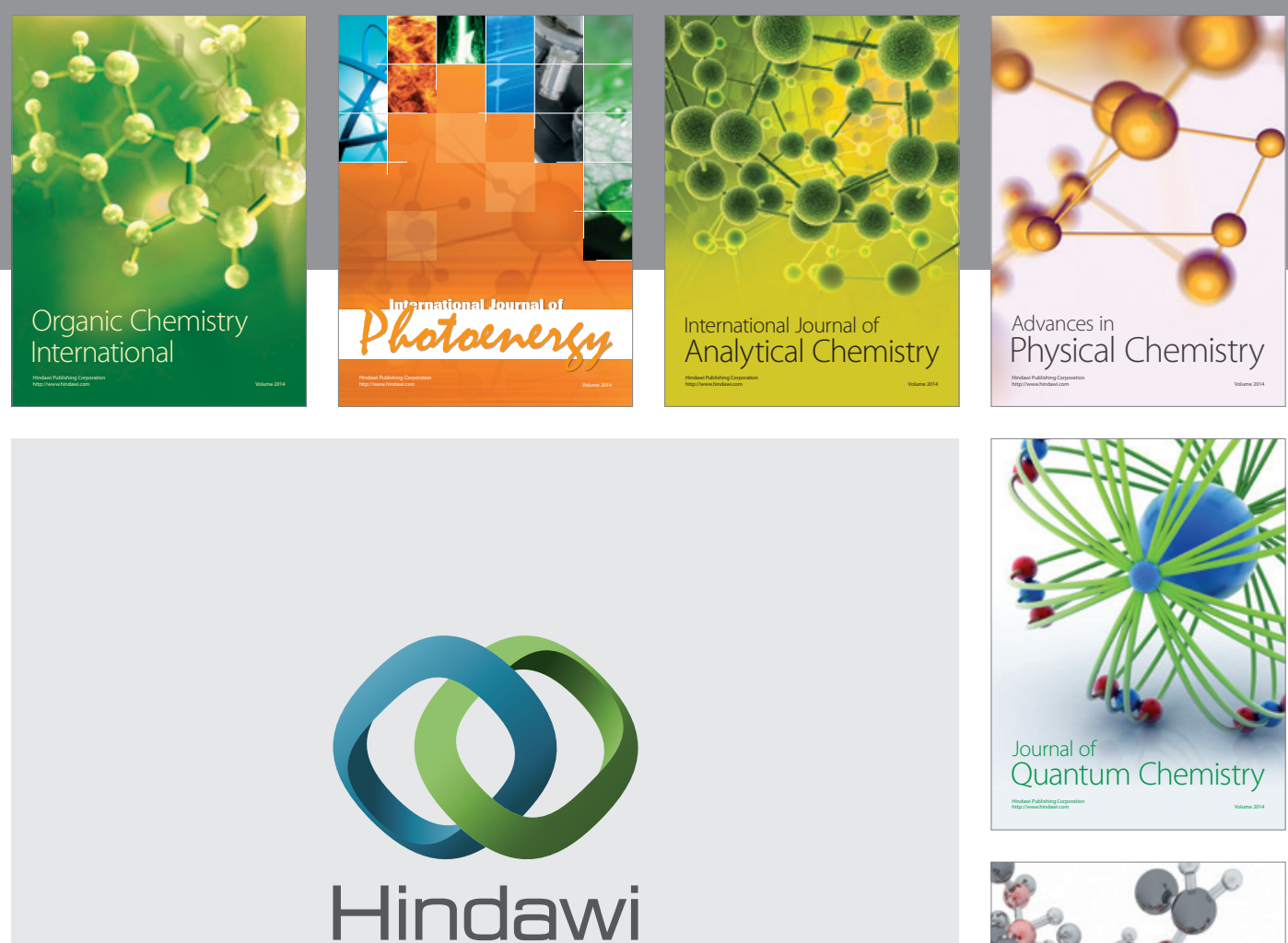

Submit your manuscripts at

http://www.hindawi.com

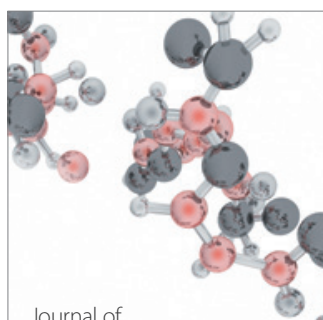

Analytical Methods

in Chemistry

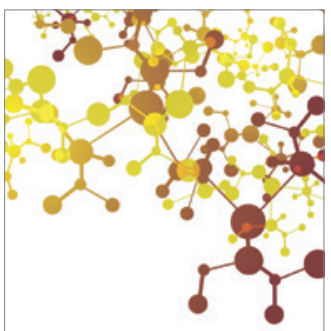

Journal of

Applied Chemistry

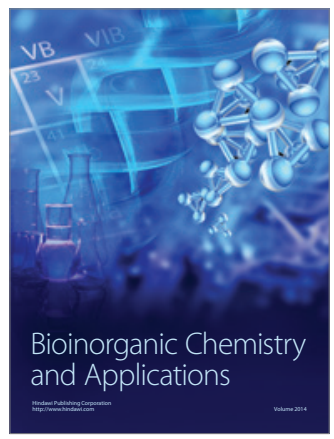

Inorganic Chemistry
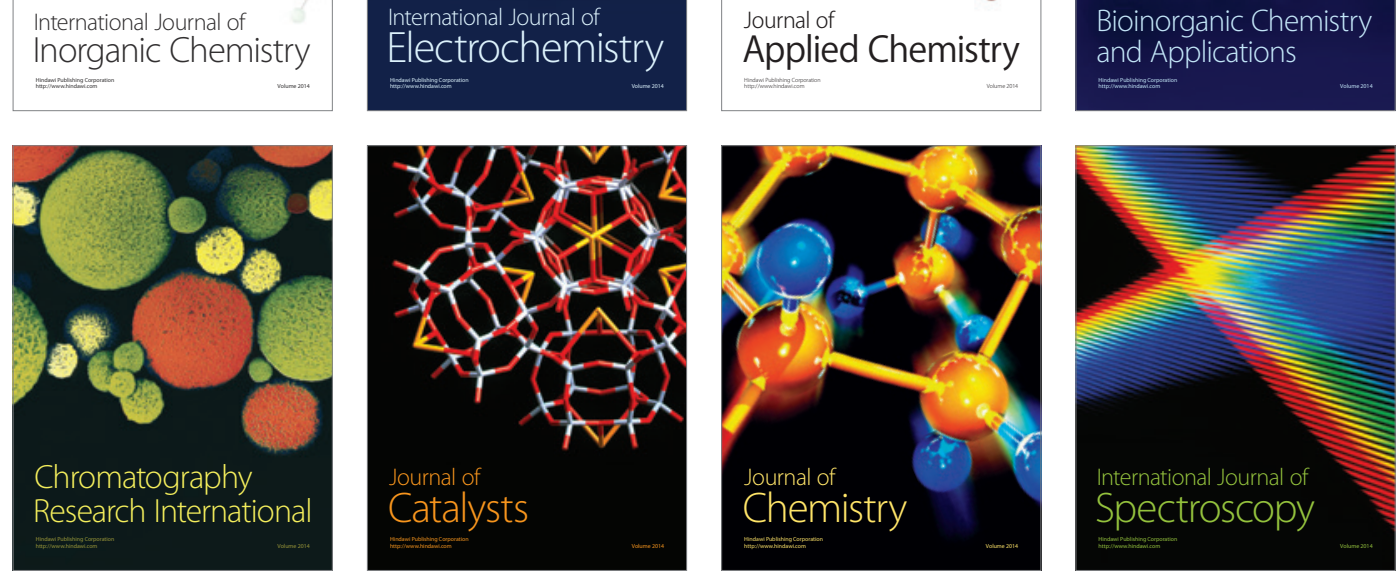